\title{
A decade of Medicare: the contribution of Private Practice Dietitians to chronic disease management and diabetes group services
}

\begin{abstract}
Aim: To review changes in utilization of dietetics services through the Medicare Chronic Disease Management program over the last decade, and describe patient uptake in 2013. Methods: Dietetics service data were extracted from published Medicare statistics for the periods (i) January 2004 to December 2013 and (ii) January to December 2013. Data comprised individual dietetic services by state and patient demography, and group services data for provider professions regarding type 2 diabetes: dietitians, diabetes educators and exercise physiologists. T-test was used to investigate the association of dietetics' individual service utilisation and workforce statistics.
\end{abstract}

Results: Individual dietetics Chronic Disease Management consultations in private practice have increased annually since 2004. Dietetics has remained the third largest provider. In 2013, a total of 302,910 individual consultations were conducted; $7 \%$ of allied health consultations. Likewise, individual services for Indigenous Australians increased since 2008. Utilization of group services for type 2 diabetes comprised $<2 \%$ of dietetics services. Dietitians provided more group services than diabetes educators but considerably fewer than exercise physiologists. Middle-aged and older patients were common, with highest uptake by those aged 55-74 years. Overall, total and per capita utilization rates were considerably higher in NSW, Victoria and Queensland compared to less populous states, although this disparity has reduced since 2010. 
Conclusions: As ten years has elapsed since the program's inception, further evaluation of the policy is needed to examine large variations in dietetics’ Chronic Disease Management uptake by state and territory in both individual and group services.

Keywords: allied health; chronic disease management; health policy; primary care 
Manuscript

\section{INTRODUCTION}

Since its inception in 2004, the Medicare Chronic Disease Management (CDM) program has enabled eligible patients to receive subsidized services from a range of allied health professionals operating in Australian primary care settings. ${ }^{1}$ The program aims to increase access to multidisciplinary care for patients living with chronic medical conditions. Based on the premise that patients who actively self-manage chronic medical conditions will limit or postpone further deterioration in their health, ${ }^{2}$ medical professionals working in teams can positively influence patient outcomes. ${ }^{3}$ There are 13 participating allied health professions in the CDM program. They are: aboriginal health workers, audiologists, chiropractors, diabetes educators, dietitians, exercise physiologists, mental health workers (including social workers or mental health nurses), occupational therapists, osteopaths, physiotherapists, podiatrists, psychologists, and speech pathologists. ${ }^{4}$

Eligible patients can access up to five subsidized individual consultations per calendar year from any of the registered allied health professions. ${ }^{5}$ Indigenous Australians can access an additional five consultations annually. In addition, patients with type 2 diabetes can be assessed for suitability and access up to eight group education sessions provided by dietitians, diabetes educators or exercise physiologists. Although the fee for each consultation is set by individual providers, they can bulk bill at the reduced rate of the Medicare scheduled fee (no out-of-pocket cost to the patient), or charge a higher rate with the rebate claimed by the patient. General Practitioners (GPs) initiate and manage each referral through collaboratively preparing a GP Management Plan and a Team Care Arrangement with the patient and determining applicable allied health disciplines. ${ }^{5}$ Further details of the CDM program and the service items are available at: http://www.medicareaustralia.gov.au. 
After a decade of operation, few studies have evaluated the CDM program. Early descriptive studies reported a lack of collaboration and communication between health professionals, particularly between general practices and allied health providers. ${ }^{6-9}$ There was recognition of a need to encourage GPs to engage with the program's team-based management approach to patient care, which differs from the traditional model of independent care provided by GPs. ${ }^{2,10}$ The challenge of facilitating teamwork was described in the NSW Team-link study, ${ }^{10}$ which found Practice Nurses vital to team communications. Overall, these studies highlight opportunities for improvement in the CDM program through evaluating its implementation.

Nationwide, utilization of the CDM program has increased annually for most allied health professions. ${ }^{11}$ Along with increases in the size of the dietetics workforce, ${ }^{1}$, dietitians registered to provide Medicare services increased to approximately 1,630 in $2013 .{ }^{13}$ Between 2004-2010, dietetics remained the third most utilized CDM profession after podiatry and physiotherapy. ${ }^{14}$ Patient uptake of individual dietetics consultations increased three-fold from 2005 (64,000 services), to 2010 (205,000 services). However, in 2010 the utilization of services across all allied health professions varied widely by geographic region ${ }^{11}$ suggesting that patients' access to local services may not be adequate across all areas of Australia. ${ }^{6}$ For example, a NSW case study of six rural general practices observed that dietetic consultations were provided at approximately half the average rate for NSW in 2005-6 and in 2006-7. ${ }^{15}$

Given that 10 years have elapsed since the inception of the CDM program, it is timely to explore the services provided by dietitians working in private practices. Understanding the rates and trends of dietetic service provision within the CDM program will inform private practice dietitians about possible service gaps and help identify opportunities for service improvement. This study aimed to review the utilization of individual and group dietetic 
services provided through the Medicare CDM program, and describe changes in utilisation over the last decade.

\section{METHODS}

Published statistics compiled from national billing data were downloaded from the Medicare Australia website at: http://www.medicareaustralia.gov.au. Based on items from the Medicare Benefits Schedule, ${ }^{4}$ dietetics service data were extracted for the periods of (i) January 2013 to December 2013 and (ii) the decade January 2004 to December 2013. Data comprised individual dietetic services (M3; item 10954) by state and by patient demographic information (age range, sex) and government expenditure. Per capita reports were extracted comprising consultations cross-tabulated with the number of individuals enrolled with Medicare in the same year. Data were extracted for the provider professions for group services for type 2 diabetes: dietitians, diabetes educators and exercise physiologists. These were assessment consultations (M9; Items 81100, 81110 and 81120) and small group education sessions (M9; items 81100 to 81125). In addition, dietetics and other service provider data for follow-on consultations for indigenous Australians were extracted (M11; Items 81300 to 81360 ). These data report the number of consultations billed to Medicare in the period rather than individual patient referral or diagnosis, which were not collected by Medicare. Data were presented using inferential statistics using IBM- SPSS version 22 (IBM. Armonk New York: 2011). The association of dietetics' individual service utilisation and workforce statistics was explored using Pearson product-moment correlation co-efficient and a significance level of $p<0.05$.

\section{RESULTS}

\section{Individual dietetics consultations}

In 2013, a total of 302,910 individual dietetics consultations were conducted. This equated to $7 \%$ of the 4.44 million consultations provided by allied health professionals 
nationally. Dietetics was the third largest provider of individual services, even though other professions had considerably higher utilization rates. Podiatry provided 2.1 million consultations and physiotherapy 1.3 million while other provider professions, considered together, conducted only 0.75 million (1.7\%) consultations. Based on the Medicare Benefits Schedule rebate for dietetics consultations of AU\$52.95, individual dietetics consultations attracted AU\$16.2 million of federal funding of the total AU\$237 million for these services.

Table 1 displays the number of individual dietetics consultations provided for each state in Australia and the rate of utilization per capita. NSW, Victoria and Queensland were the states with highest uptake, while Tasmania, ACT and NT recorded low utilization: $1 \%$ or less of total consultations. This pattern remained when investigated on a per capita basis. The less populous states/territories were potentially underserved with low consultation rates of half or less than those in more populated states or territories.

Insert Table 1 about here: Number of individual dietetics consultations provided and per capita utilisation

Service utilization was related to the geographic distribution of dietitians/dietetic clinics. There was a strong correlation between the number of individual CDM consultations provided in each state and territory in 2013 and the number of dietitians registered with Medicare by region ${ }^{13}(r=.97 ; p=<.001)$. For example, dietitians registered with Medicare in NT and in Tasmania numbered only 7 and 15 respectively, while Victoria, Queensland and NSW numbered at least 380 each.

Figure 1 displays the demographic characteristics of patients according to the total number of individual dietetics consultations in 2013. Approximately $60 \%$ of all consultations were provided to female patients. The age range of patients was between 0 -85 years and the 
age group that utilized the most consultations was 55-64 years for both sexes, comprising half of all individual dietetics consultations. Patients aged over 65 years comprised 31\% of male consultations and $27 \%$ of female consultations. Infants and children aged less than 14 years comprised only $2.5 \%$ of all consultations.

Insert Fig 1 about here: Age and gender characteristics of patients accessing individual dietetics services in 2013.

A decade of Medicare: uptake of individual consultations

Individual dietetics consultations have continued to increase over the ten years since inception of the CDM program. The annual increase in dietetics items over each of the last five years was approximately 11\%. There were 184,028 consultations in 2009 increasing to 302,970 in 2013 , comprising up to 17,000 consultations per calendar quarter.

Insert Fig 2 about here: Quarterly individual consultation rates for the main allied health professions 2004-2013.

Utilization rates of the major allied health professions increased annually. Figure 2 displays the changes in rates of utilization for the professions most utilized: podiatry, physiotherapy and dietetics. In addition, as displayed, exercise physiologists recently increased to be the fifth most utilized profession (after chiropractic services, not shown), and diabetes educators were utilized less (as shown), rating seventh after chiropractic and speech pathology (not shown).

\section{Allied health services for Indigenous Australians}


Since late 2008, additional follow-on allied health services for people of an Aboriginal or Torres Strait Islander origin have been available under similar referral conditions to individual services. This allows for up to five extra consultations in one year, with a fee schedule as outlined for individual consultations. In 2013, dietitians provided 1549 consultations ( $<1 \%$ of the total 19,500 ) with nearly all utilized (in reducing order) in NSW, Queensland and NT. Utilization patterns followed that of provider professions for individual services, with podiatry and physiotherapy the major providers.

For dietetics, the patient age span was from birth to 84+ years, however the majority (58\%) were aged between 35 - 64 years and females utilized the program in the ratio of two to one male. Since 2010, 2,975 consultations were conducted (10\% of the total), with half being provided in Queensland.

\section{Group services for people with type 2 diabetes}

Patients with type 2 diabetes referred through a GP Management Plan for small group services are initially assessed for suitability, undertaking a 45-minute face-to-face consultation with a dietitian, a diabetes educator or an exercise physiologist. The scheduled fee for providers is AU\$67.90. Patients can attend up to eight group meetings (each of at least one hour) annually with the scheduled fee in June 2014 being $\$ 19.90$ per attendance, with rebate of \$16.95 from Medicare.

Table 2 displays the number of diabetes assessment consultations and small group education sessions conducted in 2013 for the provider disciplines. During this time, dietitians provided 1718 diabetes assessments and 2840 small group education sessions. Most of these services were utilised by patients in NSW, who attended $68 \%$ of dietetic assessments and $62 \%$ of group dietetics services. Group dietetics services in SA, WA, Tasmania, ACT and NT were very limited. 
Insert Table 2 about here: Group services for type 2 diabetes: Number of assessment items and education items conducted in 2013 by state/territory.

Service utilisation was related to the geographic distribution of dietitians registered to provide group services (amounting to 2/5 of clinics). There was a strong correlation between the number of assessment consultations conducted by dietitians in each state and territory in 2013, and the number of Medicare-registered group services dietitians by region $(\mathrm{r}=.714 ; \mathrm{p}=$ .047). Dietitians' total group services comprised $<2 \%$ of all their Medicare CDM service provision. Although dietitians conducted more diabetes assessments and education sessions than diabetes educators, the group services of exercise physiologists were utilised to a larger extent than dietetics or diabetes educators combined. For example, in NSW, exercise physiologists provided nine times more group education sessions than dietitians. It should be noted that patients with type 2 diabetes would also be eligible to consult with dietitians, diabetes educators and/or exercise physiologists through individual CDM consultations as described earlier.

Each patient utilizing group education sessions undergoes an assessment, with this data providing a cross-sectional picture of patient characteristics. Females and males accessed dietetics assessment services approximately equally. The most common age range for both sexes was 55 - 64 years, with patients aged 55 - 74 comprising half of all referrals (55\%). The age span was from 15 years to more than 85 years although very few $(<1 \%)$ patients were young adults aged $\leq 34$ years or were aged $\geq 85$ years.

Insert Figure 3 about here: Quarterly utilization of small group education sessions for patients with type 2 diabetes between 2007-13 for dietetics, diabetes educators and exercise physiology. 
Utilization of group services for type 2 diabetes 2007-2013

Figure 3 displays the changes in rates of utilization for group education sessions for dietitians, diabetes educators and exercise physiologists between inception in 2007 and December 2013. Dietetics education sessions peaked at almost 5,200 in 2011, but declined by $20 \%$ to 4,400 in 2012 and by $46 \%$ to 2,800 in 2013. Diabetes educator sessions showed a similar pattern, with 2070 sessions conducted in 2013. In contrast, exercise physiology sessions have continued to escalate (35,400 in 2013).

\section{DISCUSSION}

This study reviewed the utilization of individual and group dietetics services in private practice through the Medicare CDM program over the decade 2004 - 2013. The use of individual dietetics services increased consistently although there was wide variation in the rates of service provision across Australia. The use of group dietetics services for type 2 diabetes has remained low and has decreased over recent years.

The most recent review of Medicare CDM dietetics services occurred in $2010 .{ }^{14}$ Since this time, the annual number of individual dietetics services has increased by over $150 \%$ : from 925 consultations per 100,000 in 2010, to 1293 consultations per 100,000 in 2013. The greatest change in service provision has occurred in less populous states and territories, including ACT, Tasmania and the NT, which increased by $428 \%, 230 \%$ and $205 \%$ respectively since 2010. Thus the disparity in service provision across the states and territories still exists, but is declining.

A potential barrier to service provision raised in the past has been a lack of available allied health professionals to provide CDM services, particularly in rural areas and in less populous states. ${ }^{11,15}$ Recent workforce data tends to confirm this association. Populationbased data shows fewer allied heath professionals employed as the distance from major urban 
centres increases, including dietitians, physiotherapists and podiatrists. ${ }^{12,16,17}$ Health Workforce Australia 2011 data reported on average 10.3 dietitians were employed in major cities per 100,000 population, 7.7 in inner regional centres, 6.6 in 'remote' areas and 2.7 in 'very remote' areas (eg, the NT or parts of Queensland and WA). ${ }^{12}$ Thus, allied health accessibility is likely to also form a barrier to patient referrals for chronic disease management in some primary care settings. Nevertheless, there has been an average of $50 \%$ increase in dietitians recorded across all remoteness categories between 2006 and $2011 .^{12}$ Cultural differences may impact provision of CDM consultations to Indigenous Australians and with the increasing presence of accredited practising dietitians who have an Indigenous heritage ${ }^{13}$ there will be opportunity for these services to increase further.

Dietetics has remained the third most utilised profession for individual consultations over the last decade. With the number of dietitians working in primary care also increasing over this period ${ }^{16}$ it follows that the CDM program may provide an incentive for dietitians to commence this work. The latest workforce survey to profile private practice dietitians indicated that of 151 dietitians, 94\% were registered with Medicare, half operated in a general practice setting and half reported their main source of referral as GPs. One-third (41\%) perceived themselves as not receiving adequate remuneration for private practice work, ${ }^{18}$ also identifying several barriers to best practice in chronic disease management. ${ }^{19}$ For example, there is no remuneration available for dietitians to communicate with or meet with other team members to review team-based care and/or plan patient management. Under the Medicare fee structure of remuneration for consultations of at least 20 minutes, dietitians express they are not funded by Medicare to complete the pre-visit administration and post-visit reporting tasks required. ${ }^{19}$ Nevertheless, dietetics services operating within the constraints of the CDM program are consistently increasing, suggesting the enablers to dietitians providing services 
through the CDM program outweigh the barriers to best-practice chronic disease management.

Utilisation of group services for type 2 diabetes provided by dietitians has remained relatively low. Optimally, Medicare-subsidized diabetes education programs are multidisciplinary. ${ }^{20}$ Group education sessions may facilitate participants' self-efficacy and empowerment through peer learning and sharing, supporting goal setting and problem solving skills. ${ }^{21}$ Dietitians were contributing to the assessment of patients to commence group education although they were not conducting many group sessions themselves. There was a disparity between the number of dietitians' and diabetes educators' assessments and small group sessions compared with the more dominant exercise physiologists who provided almost $90 \%$ of all group services. Reasons for this difference were unclear. Patients with diabetes may have ready access to public hospital clinics or other specialist diabetes education services where a dietitian is situated. A study of rural and remote service provision for people with diabetes by registered nurses and Aboriginal health workers in NSW noted service duplication with confusion about funding. ${ }^{22}$ Only two of every five dietetics clinics were providers of group services, ${ }^{13}$ often working as sole part-time practitioners ${ }^{\mathbf{1 9}}$ and thus accessibility of dietitians was likely reduced. Alternatively, however, exercise physiologists are almost exclusively private practitioners with an increasing presence. ${ }^{23}$ Dietitians who conduct CDM group education sessions have previously reported a lack of access to appropriate facilities and to multidisciplinary providers as barriers to collaborative working. ${ }^{19}$ These and other factors may prevent dietitians from being involved in further development of group programs for patients with type 2 diabetes. Development of a common national curriculum may help resolve these issues.

Despite a continued increase in the overall utilisation of CDM services, minimal evaluation has occurred regarding patient outcomes. One prospective observational study 
conducted over nine months found modest changes in weight and waist circumferences for CDM patients with type 2 diabetes. ${ }^{24}$ However, there was a high rate of non-attendance (29\%) over time. Another recent qualitative investigation found that some CDM patients were motivated by feeling an obligation to attend consultations with allied health professionals due to the rebates available to them. ${ }^{25}$ This suggests that other determinants are influencing patients' attendance and adherence and further research is necessary to more fully evaluate program delivery and outcomes.

The present study has some limitations. Although valuable, national indicator data is limited because it does not allow full interpretation of referred patient characteristics. For example, it is not known how many patients were referred through the CDM program, the number of consultations each patient attended, or valid gender comparisons. Regardless, the current report provides a snapshot of national CDM dietetics service levels both for individual consultations and for group services which may help dietitians to identify opportunities for service improvement.

\section{CONCLUSION}

Utilization of Medicare CDM referrals is increasing at a rapid rate, with dietetics experiencing $11 \%$ growth in individual consultations over each of the last five years. Dietetics remains the third largest provider of individual services, and the disparity in service utilization between states and territories is decreasing. As group dietetics services comprises $<2 \%$ of total dietetic service provision and the rate of group education sessions has decreased, there is scope for dietitians to be more involved in collaborative small group diabetes care. Little is known about large variations in dietetics CDM uptake by state and territory and this warrants further exploration if dietitians are to continue to be key providers for Medicare chronic disease management in the future. 


\section{References}

1. The Department of Health. Chronic disease management VCDM items. 2013.Available at: http://www.health.gov.au/internet/main/publishing.nsf/Content/mbsprimarycarechronicdiseasemanagement. 2013 Accessed 4 May 2014.

2. Dennis S, Zwar N, Griffiths R et al. Chronic disease management in primary care: from evidence to policy. Med J Aust 2008; 188: S53-6.

3. Wagner EH, Glasgow RE, Davis C et al. Quality improvement in chronic illness care: a collaborative approach. Joint Com J Qual Improv 2001; 27: 63-80.

4. Australian Government Department of Health. Medicare benefits schedule book: Category 8. Available at: http://www.health.gov.au/mbsonline accessed 6 January 2014.

5. MBS Online Medical Benefits Schedule 2014 Available at: http://www.health.gov.au/internet/mbsonline/publishing.nsf/Content/Medicare-Benefits-ScheduleMBS-1 accessed 22 March 2014.

6. Foster $\mathrm{M}$, Cornwell $\mathrm{P}$, Fleming $\mathrm{J}$ et al. Better than nothing? Restrictions and realities of enhanced primary care for allied health practitioners. Aust J Primary Health 2009; 15: 326-34.

7. Middlebrook S, Mackenzie L. The Enhanced Primary Care program and falls prevention: Perceptions of private occupational therapists and physiotherapists. Australas J Ageing 2012; 31: 727. DOI: 10.1111/j.741-6612.2011.00527.x

8. Skeat J, Morgan A, Nickless T. Playing by the rules: speech pathologists' views about patient suitability for the Enhanced Primary Care Program. Aust Fam Phys 2009; 38: 269-72.

9. Cant R. Patterns of delivery of dietetic care in private practice for patients referred under Medicare Chronic Disease Management: results of a national survey. Aust Health Rev 2010; 34: 1-7. DOI: 10.1071/AH09724

10. Harris M, Chan B, Daniel C et al. Development and early experience from an intervention to facilitate teamwork between general practices and allied health providers: the Team-link study. BMC Health Serv Res 2010: 104. doi:10.1186/472-6963-10-104

11. Cant R, Foster M. Investing in big ideas: Utilization and cost of Medicare Allied Health services in Australia under the Chronic Disease Management initiative in primary care. Aust Health Rev 2011; 35: 468-74. http://dx.doi.org/10.1071/AH10938 
12. Health Workforce Australia. Australia's health workforce series - Dietitians in focus.

HWA; Adelaide: 2014. At: hwa.gov.au Accessed 10th May 2014.

13. Dietitians Association of Australia. Annual report 2013. Canberra, DAA: 2013. Available at: http://daa.asn.au Accessed 30th May 2014.

14. Cant R, Foster M. Update on Medicare-subsidized Allied Health Chronic Disease Management consultations: Dietetics \& the five most referred professions, 2010. Nutr Diet 2012; 70: 42-8. DOI: 10.1111/j.747-0080.2012.01635.x

15. Brown L, Mitchell L, Williams L, Macdonald-Wicks L, Capra S. Private practice in rural areas: An untapped opportunity for dietitians. Aust J Rural Health 2011; 19: 191-6.

16. Health Workforce Australia. Australia's health workforce series - Physiotherapists in focus. HWA; Adelaide: 2014b. At: hwa.gov.au Accessed 10th May 2014.

17. Health Workforce Australia. Australia's health workforce series - Podiatrists in focus. HWA; Adelaide: 2014c. At: hwa.gov.au Accessed 10th May 2014.

18. Ball L, Larsson R, Gerathy R, Hood P, Lowe C. Working profile of Australian private practice Accredited Practising Dietitians. Nutr Diet 2013; 70: 196-205.

19. Jansen S, Ball L, Lowe C. Impact of the Medicare Chronic Disease Management Program on the conduct of Australian dietitians' private practices. Aust Health Rev 2014; under review

20. O’Brien, S. Private practice diabetes education: What role does it have in providing a holistic model of diabetes care in far North Queensland, Australia. J Diabetes Metab 2013; 4: 6. At http://dx.doi.org/10.4172/2155-6156.S1.022.

21. Lawn S, Schoo A. Supporting self-management of chronic health conditions: common approaches. Patient Educ Couns 2010; 80: 205-11.

22. King M., King L, Willis E, Munt R, Semmens, F. Issues that impact on Aboriginal Health Workers' and Registered Nurses' provision of diabetes health care in rural and remote health settings. Aust J Rural Health 2013; 21: 306-12. doi: 10.1111/ajr.12062

23. Cheema B, Robergs R, Askew C. Exercise physiologists emerge as allied healthcare professionals in the era of non-communicable disease pandemics: A report from Australia, 20062012. Sports Med 2014; 44: 869-77. doi: 10.1007/s40279-014-0173-y 
24. Spencer L, OShea M-C, Ball L, Desbrow B, Leveritt M. Attendance, weight and waist circumference outcomes of patients with type 2 diabetes receiving Medicare-subsidised dietetic services. Aust J Primary Health 2013: 20: 291-7. doi.org/10.1071/PY13021

25. Foster M, Mitchell GK. 'The onus is on me': primary care patient views of Medicare-funded team care in chronic disease management in Australia. Health Expectations 2013;

doi:10.1111/hex.12061 